\title{
Publisher Correction: Integrating uncertainty into public energy research and development decisions
}

Laura Díaz Anadón, Erin Baker and Valentina Bosetti

Correction to: Nature Energy https://doi.org/10.1038/nenergy.2017.71 (2017); published online 9 May 2017.

In the version of this Review originally published, the received and accepted dates were omitted; they are 25 July 2016 and 29 March 2017, respectively. These errors have now been corrected in all versions of the Review.

Published online: 22 November 2017

https://doi.org/10.1038/s41560-017-0052-5 